\title{
The effect of surface nano-corrugation on the squeeze-out of molecular thin hydrocarbon films between curved surfaces with long range elasticity.
}

\author{
I.M. Sivebaek ${ }^{1,2,3}$ and B.N.J. Persson ${ }^{1,4}$ \\ ${ }^{1}$ IFF, FZ-Jülich, 52425 Jülich, Germany \\ ${ }^{2}$ Novo Nordisk , Device R\&D, 3400 Hillerød, Denmark \\ ${ }^{3}$ Mechanical Engineering, Technical University of Denmark, 2800 Lyngby, Denmark \\ ${ }^{4}$ MultiscaleConsulting.com
}

\begin{abstract}
The properties of linear alkane lubricants confined between two approaching solids are investigated by a model that accounts for the roughness, curvature and elastic properties of the solid surfaces. We consider linear alkanes of different chain lengths from $\mathrm{C}_{3} \mathrm{H}_{8}$ to $\mathrm{C}_{16} \mathrm{H}_{34}$, confined between corrugated solid walls. The pressure necessary to squeeze out the lubricant increases rapidly with the alkane chain length, but is always much lower than in the case of smooth surfaces. The longest alkanes form domains of ordered chains and the squeeze-out appear to nucleate in the more disordered regions between these domains. The short alkanes stay fluid-like during the entire squeeze out process which result in a very small squeeze-out pressure which is almost constant during the squeeze-out of the last monolayer of the fluid. In all cases we observe lubricant trapped in the valley of the surface roughness, which cannot be removed independent of the magnitude of the squeezing pressures.
\end{abstract}

PACS numbers:

\section{INTRODUCTION}

Lubricants are used in many mechanical devices to reduce friction and wear. Many lubricants consist of a base oil, usually a hydrocarbon fluid, with additives. The additives usually form protective coatings on the solid walls. If the base oil is squeezed out from the contact regions, the adsorbed film, at least if in a (twodimensional (2D)) solid-like state, can reduce the friction and the wear. However, even long-chain hydrocarbons can adsorb on solid walls and form protective coatings which can survive relative high contact pressures without getting removed[1, 2]. Thus in Ref. [2] we have shown that adsorbed $\mathrm{C}_{14} \mathrm{H}_{30}$ films between smooth surfaces may for short time withstand contact pressures approaching $1 \mathrm{GPa}$, which is similar to the plastic yieldstress of steel. In the present paper we will extend the study of [2] and present results for solid walls with periodic short-wavelength nano-roughness. We note that this study, and that in [2], involve fluid squeeze-out between a curved surface and a nominally flat surface. This differs from our earlier (frictional) studies involving flat surfaces [3-6].

We are concerned about the squeeze-out of lubricant between rough surfaces until dry contact is reached[7]. The squeeze-out of liquids has been studied using the Atomic Force Microscope (AFM)[8-11] and the Surface Force Apparatus [12-14]. In the former case it has been established that huge wear occurs when the last lubricant layer has been squeezed out.

A common method to study wear is shown in the insert of Fig. 1. Here a steel ball is slid in reciprocating motion on a lubricated steel disk under standard conditions $[15,16]$. The resulting wear scar diameter on the ball expresses the lubricity of the tested lubricant. This set-up has been used to test the lubricity of a series of alkanes [17]. Fig. 1 shows the wear scar diameter measured

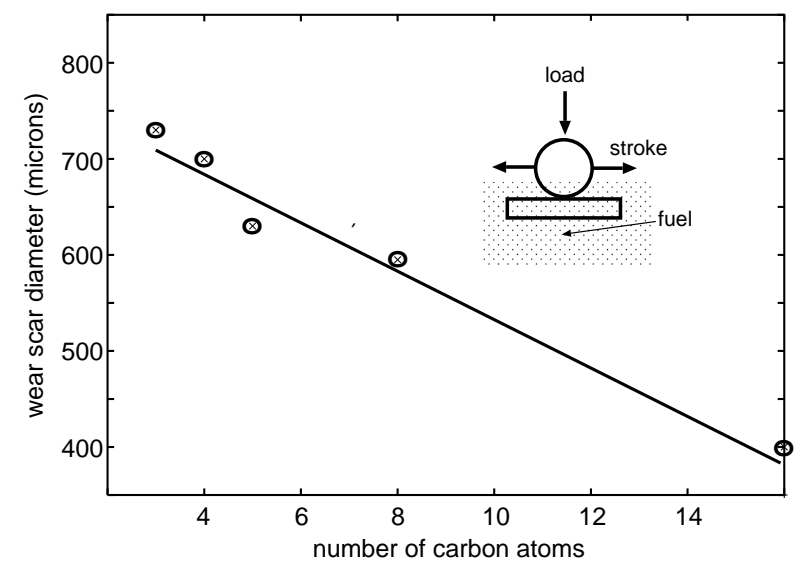

FIG. 1: The wear expressed as the wear scar diameter as a function of the chain length of the hydrocarbon. The insert shows the ball on disk configuration in a wear test rig. Adapted from [2].

on the ball as a function of the hydrocarbon molecular length [2]. Note that the wear volume depends non-linear on the wear scar diameter $D$ as $V \approx \pi D^{4} /(64 R)$, where $R$ is the radius of the ball. Hence the wear volume depends highly non-linearly on the alkane chain length and the wear volume is $\sim 11$ times higher when $\mathrm{C}_{3} \mathrm{H}_{8}$ is used as lubricant as compared to $\mathrm{C}_{16} \mathrm{H}_{34}$.

Fig. 1 shows that the longer alkanes have higher lubricities than the shorter ones, suggesting that the longer chain molecules are better boundary lubrication films. However, the viscosity also increases with the chain length so an influence of this (bulk) property on the wear scar diameter could not be excluded.

Using a molecular dynamics model that accounts for the curvature and long range elasticity of surfaces [18], 
the influence of the alkane length on the lubricity has been investigated [2]. It appeared that longer alkanes are capable of including more lubricant atoms in the contacts than shorter ones. This results in a higher binding energy per unit surface area, which makes them better boundary lubricants. However, the effect was not so strong and the pressure to nucleate squeeze-out only increased with a factor of $\sim 2$ as the chain length increased from $\mathrm{C}_{3} \mathrm{H}_{8}$ to $\mathrm{C}_{16} \mathrm{H}_{34}$.

The theoretical study [2] investigated smooth surfaces whereas the steel surfaces in wear tests supposedly have roughness on many length scales. The purpose of the present study is to clarify the role of surface corrugation in lubricant layer transitions and wear. We will also propose a theory which can explain the molecular dynamics simulation results. When including the short-range nanocorrugation we find in this study a much larger variation in the pressure to nucleate squeeze-out, which increases with a factor of $\sim 20$ as the chain length increases from $\mathrm{C}_{3} \mathrm{H}_{8}$ to $\mathrm{C}_{16} \mathrm{H}_{34}$.

\section{THE MODEL}

The model is described in Refs. [2, 18, 19], but we review it briefly here. We are concerned with the properties of a lubricant film squeezed between the curved surfaces of two elastic solids. In experiments, a system of this type is obtained by gluing two elastic slabs (of thickness $W_{1}$ and $W_{2}$ ) to "rigid" surface profiles of arbitrary shape. If the radii of curvature of the rigid surfaces are large compared to $W_{1}$ and $W_{2}$, the elastic slabs will deform, reproducing with their free surfaces the (nearly arbitrary) shape of the underlying rigid profiles.

In what follows we denote the lower solid as substrate, which is taken to be fixed in space. The upper solid, denoted as block, will be moving. To account for the elastic response of the slabs, without dealing with the large number of atoms required to simulate a mesoscopic elastic solid we treat explicitly, at the atomistic level, only the last atomic layer of the solids at the interface. These atoms are connected to a rigid curved surface (or profile). The force constants connecting these atoms to the rigid profile, however, are not the bare parameters, determined by the model interatomic potential. Instead, those force constants are treated as effective parameters that implicitly re-introduce the elastic response of the slabs of arbitrary width $W_{1}$ and $W_{2}$.

The model is illustrated in Fig. 2 (see also Refs. $[2,18]$ ). The atoms in the bottom layer of the block (open circles) form a simple square lattice with lattice constant $a$, and lateral dimensions $L_{x}=N_{x} a$ and $L_{y}=N_{y} a$. In the following, periodic boundary conditions are assumed in the $x y$ plane. The atoms interact with each other via "stiff" springs (thick lines) and execute bending and stretching motion characterized by a bending force constant $k_{0 B}$ and a stretching force constant $k_{0}$, respectively. Moreover, each atom is connected to the upper rigid sur-

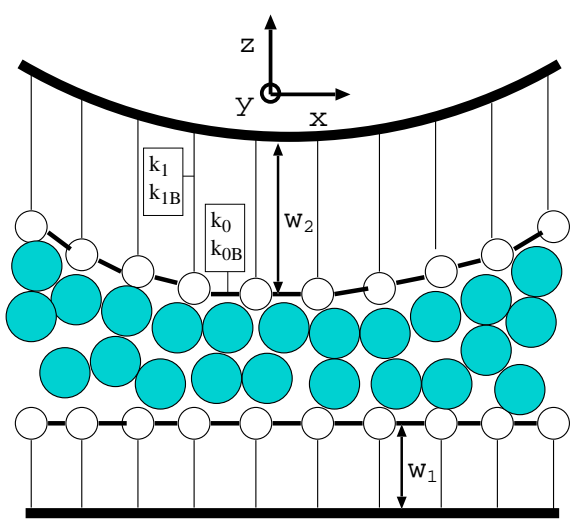

FIG. 2: The model used in this study (schematic). The white circles are the block and substrate surface atoms and the colored ones are lubricant atoms or molecules[18]. The slab thicknesses W1 and W2 are also shown together with axes indicating the $\mathrm{x}, \mathrm{y}$ and $\mathrm{z}$ directions.

face profile by "soft" elastic springs (thin lines), of bending force constant $k_{1 B}$ and stretching force constant $k_{1}$. As described in Refs. [2, 18], the numerical values of all these force constants $k_{0}, k_{0 B}, k_{1}$ and $k_{1 B}$ are determined in such a way as to mimic the elastic response of the entire slab.

The substrate is treated in a similar way as the block, but we use slightly different lattice constant in order to avoid having (low order) commensurate structures formed at the interface. The space between the block and the substrate is occupied by a layer (monolayer or more) of the lubrication fluid (full circles in Fig. 2).

The MD (molecular dynamics) calculations have been performed keeping the temperature of the solid walls fixed at their outer boundaries by a Langevin thermostat with a coupling time constant of $10^{-11} \mathrm{~s}$ for both the substrate and the block. (see Ref. [18]). This is a realistic treatment, and it implies that heat flows from the lubricant to the confining walls. The walls have been thermostatted at $300 \mathrm{~K}$ except for the study investing the influence of temperature on the squeeze-out where we use temperatures between $0 \mathrm{~K}$ and $700 \mathrm{~K}$.

Below we study mainly the average pressure. The pressure acting on a wall atom is defined as the total normal force acting on the wall atom from the lubricant atoms and from the other wall, divided by the area $a^{2}$. The average pressure is the $z$-component of the total force acting on the solid block from the lubricant and the substrate, divided by the total area $L_{x} \times L_{y}$.

The elastic modulus and Poisson ratio for the block and substrate were $E=7.72 \cdot 10^{10} \mathrm{~Pa}$ and $\nu=0.42$, respectively, corresponding to the values for gold. We used systems with two types of substrates - smooth and "nano-corrugated" (see details below). In the case of the system with smooth substrate we used the same thickness for the block and substrate $W=50 \AA$. This choice of 


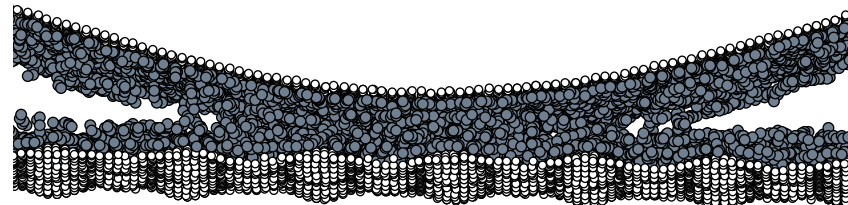

FIG. 3: The squeezing set-up with surface corrugation. The atomically corrugated block has a cosine shaped surface, and the substrate has nano-corrugation. The lubricant is octane $\left(\mathrm{C}_{8} \mathrm{H}_{18}\right)$ and the distance between the block and the substrate is $20 \AA$.

thicknesses implies that the block and the substrate used in our simulations will deform elastically similar to each other. In the case of the system with nano-corrugated substrate we used the substrate thickness $W_{1}=10 \AA$ and the block thickness $W_{2}=90 \AA$. A larger substrate thickness would make the nano-corrugation collapse rendering squeeze-out less visible. In the simulations we used a system of lateral dimensions $L_{x}=506 \AA, L_{y}=75.9 \AA$. For the substrate we used $N_{x}=200$ and $N_{y}=30$ atoms in the $x$ and $y$ directions, forming a square lattice with lattice constant $a=2.53 \AA$. The corresponding parameters for the block were $N_{x}=180, N_{y}=27$ and $a=2.81$ A.

The block rigid profile was taken to be cosine corrugated in the $x$ direction, with corrugation amplitude (difference between maximal and minimal surface heights) $0.1 L_{x}$ and wavelength $L_{x}$. We used two types of substrate corrugations - smooth surface and "nano-corrugated" surface. In the latter case the rigid substrate profile had a sine corrugation of the form

$$
h(x, y)=h_{0} \sin \left(q_{x} x\right) \sin \left(q_{y} y\right)
$$

where $q_{x}=2 \pi / \lambda_{x}$ and $q_{x}=2 \pi / \lambda_{y}$, and with the roughness amplitude $h_{0}=5 \AA$ and the wavelengths $\lambda_{x}=L_{x} / 13$ and $\lambda_{y}=L_{y} / 2$. Thus we studied the effect of nano-corrugation of the substrate on the confined lubricant structure and dynamics of squeezing.

Alkane lubricants were used in the present calculations. We considered alkane molecules of different chain lengths $\mathrm{C}_{3} \mathrm{H}_{8}, \mathrm{C}_{4} \mathrm{H}_{10}, \mathrm{C}_{8} \mathrm{H}_{18}, \mathrm{C}_{9} \mathrm{H}_{20}, \mathrm{C}_{10} \mathrm{H}_{22}, \mathrm{C}_{12} \mathrm{H}_{26}, \mathrm{C}_{14} \mathrm{H}_{30}$ and $\mathrm{C}_{16} \mathrm{H}_{34}$. We considered $\mathrm{C}_{n} \mathrm{H}_{2 n+2}$ chain molecules consisting of $n$ beads in the united atom representation. The Lennard-Jones potential was used to model the interaction between beads of different chains with relevant parameters [20-22]

For interaction between atoms of the block and the substrate we used the Lennard-Jones potential with the same parameters as for the interaction of each bead with the block and substrate atoms. We estimate this approach to be accurate enough to compare the squeeze-out pressures of alkanes of different lengths.

\section{SIMULATION RESULTS}

In this section we describe the results obtained from our simulations for eight different linear alkanes as lubricants. Two cases are investigated: One with pure squeezing and one with combined squeezing and sliding. All velocities are $1 \mathrm{~m} / \mathrm{s}$ and at the start of the squeezing the systems are in thermal equilibrium at the temperature $300 \mathrm{~K}$.

We focus mainly on the processes involved in removing the last monolayer of adsorbed alkanes, as this is most important for the wear. Fig. 3 shows the squeezing set-up with surface corrugation. The block has a cosine shaped bottom surface and the substrate surface is nominally flat with nano-corrugation. The lubricant is octane $\left(\mathrm{C}_{8} \mathrm{H}_{18}\right)$ and the distance between the block and the substrate is $20 \AA$.

Fig. 4 shows snapshot pictures of the lubricant film, after removing the substrate and the block, during squeezeout of the last monolayer. Results are shown for $\mathrm{C}_{3} \mathrm{H}_{8}$, $\mathrm{C}_{8} \mathrm{H}_{18}$ and $\mathrm{C}_{16} \mathrm{H}_{34}$, in the pressure range between the black and open circles in Fig. 5. The snapshots are numbered 1-5, the average pressure and the distance traveled by the block in each case are indicated under the snapshots.

Fig. 5 shows the average pressure as a function of the distance moved by the upper surface of the block. Results are shown for several chain lengths and for the squeezing velocity $1 \mathrm{~m} / \mathrm{s}$. The black dots correspond to the pressures and distances at which the squeeze-out of the last monolayer is initiated (initial contact), and the open circles to what we denote as full wall-wall contact. The insert of Fig. 5 shows the pressure evolution as the lubricant is squeezed out.

At the nanoscale many studies have discussed when there is actual contact between two surfaces [23-26]. In the present study, we define initial contact as the first squeeze-out of lubricant between the block and the substrate. This is observed visually by detecting holes in the lubricant film illustrated by the images with the number 2 in Fig. 4. Full wall-wall contact is obtained when there is clear squeeze-out in more corrugation points corresponding to the images 5 in Fig. 4.

Note that for the $\mathrm{C}_{3} \mathrm{H}_{8}$ case in Fig. 4, the alkane molecules are arranged in a disordered pattern on the surface, indicating a $2 \mathrm{D}$ liquid-like state. However, for the $\mathrm{C}_{16} \mathrm{H}_{34}$ system, small domains of molecules, with the molecular axis aligned in the same direction, can be observed. We refer to this state as a $2 \mathrm{D}$ solid-like state with local order (crystalline domains) but without long range order. Detailed inspection of the snapshot pictures for the $\mathrm{C}_{16} \mathrm{H}_{34}$ system shows that the squeeze-out of the last monolayer start in the regions between the crystalline domains. Clearly, these disordered domain-wall regions are the weak points with respect to squeeze-out. This is similar to the propagation of cracks in natural rubber: natural rubber undergoes strain crystallization at large strain. Thus, at high crack speed, where the strain at the 
1

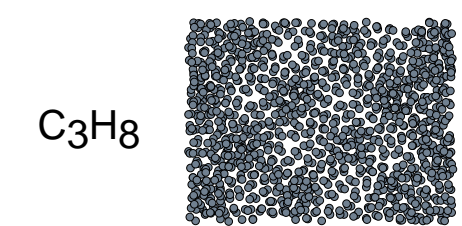

pressure (MPa) 10

distance (Ang) 12

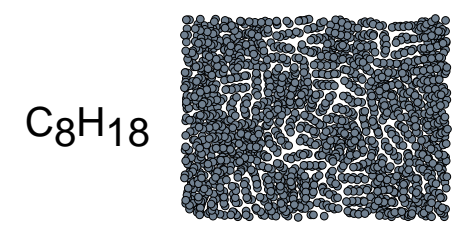

pressure (MPa) 65

distance (Ang) 12.6

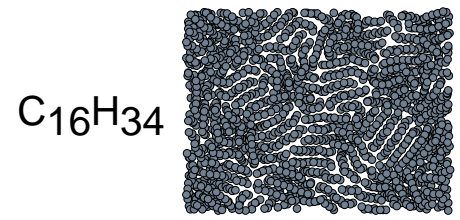

pressure (MPa) 200

distance (Ang) 14
2

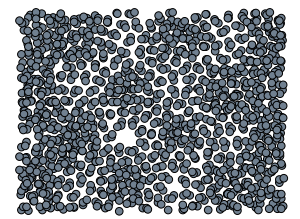

10

12.1

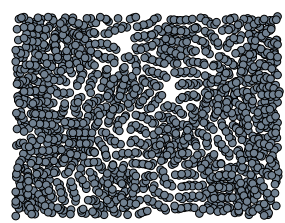

68

12.8

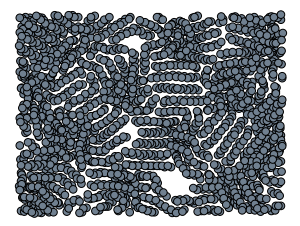

220

14.5
3

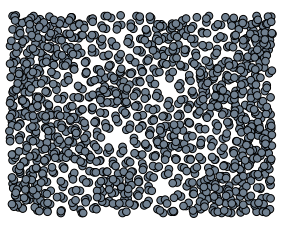

10

12.2

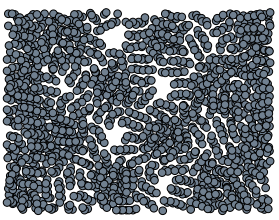

70

13

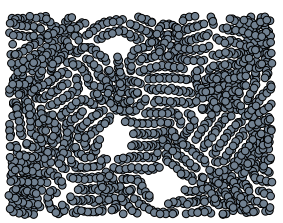

220
4

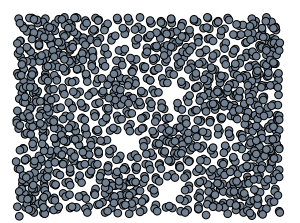

10

12.3

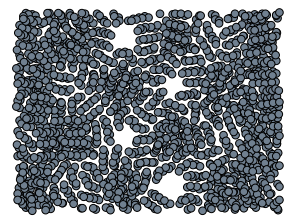

75

13.2

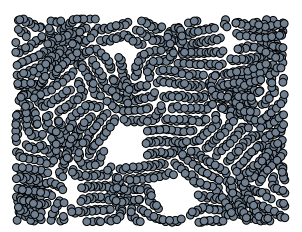

240

15.5
5

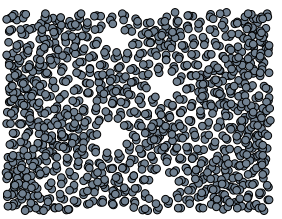

10

12.4

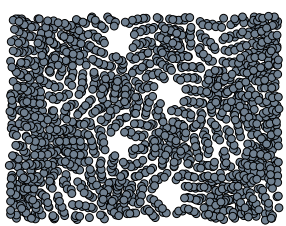

80

13.4

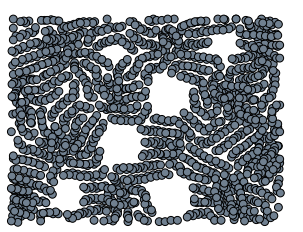

270

16

FIG. 4: Snapshots of the squeezing out of the alkanes $\mathrm{C}_{3} \mathrm{H}_{8}, \mathrm{C}_{8} \mathrm{H}_{18}$ and $\mathrm{C}_{14} \mathrm{H}_{30}$. The squeezing velocity is $1 \mathrm{~m} / \mathrm{s}$. The average pressure and the distance traveled by the block in each system are indicated under each snapshot. The snapshots are numbered $1-5$.

crack tip is very high, close to the crack tip the hydrocarbon chains form an ordered crystalline arrangements [27], which results in a strong increase in the crack propagation energy, i.e., the crystalline regions are mechanically much stronger than the disordered (amorphous-like) regions $[28,29]$.

Note the low and nearly constant pressure $p_{0} \approx$ $10 \mathrm{MPa}$ necessary to remove the $\mathrm{C}_{3} \mathrm{H}_{8}$ monolayer. We attribute this to the liquid-like nature of this adsorbed film (see discussion section). For the longer alkane chain systems much higher squeeze-out pressure is required, and the squeeze-out takes much longer time than for the $\mathrm{C}_{3} \mathrm{H}_{8}$ system. We attribute this to the solid-like nature of these lubrication films, and to the pinning barriers experienced by the monolayers. As compared to the present case, for smooth curved surfaces (see Ref. [2]) we observed much higher squeeze-out pressures, and much weaker dependency of the squeeze-out pressure on the alkane chain length. Thus, for smooth surfaces, under the same conditions as above, the (average) pressure at the nucleation of squeeze-out increased nearly linearly with the chain length from $\approx 350 \mathrm{MPa}$ for $\mathrm{C}_{3} \mathrm{H}_{8}$ to $\approx 800 \mathrm{MPa}$ for $\mathrm{C}_{16} \mathrm{H}_{34}$, i.e., $\sim 35$ times higher pressure for the $\mathrm{C}_{3} \mathrm{H}_{8}$ system than in the present case, but only $\sim 3$ times higher for the $\mathrm{C}_{16} \mathrm{H}_{34}$ system. This reflects the different physical processes involved: for the smooth surfaces the nucleation of squeeze-out involves a fluctuation (thermally activated process) where a small "hole" is first formed in the lubrication film. In this process the solid walls bend inwards into the hole, which reduces the elastic energy stored in the system. As will be shown in the discussion section, in the present case with nano-corrugation, the onset of squeeze-out is more like an indentation process in a soft solid (here alkane film).

Fig. 6 shows snapshot pictures of the $\mathrm{C}_{3} \mathrm{H}_{8}, \mathrm{C}_{8} \mathrm{H}_{18}$ and $\mathrm{C}_{16} \mathrm{H}_{34}$ systems when the upper surface of the block has moved $10 \AA$ towards the substrate after the point where the substrate and the block first mad contact in the absence of the lubricant. The islands of trapped alkanes cannot be removed independently of the magnitude of the applied pressure. Similar effects will take place when a rubber block is squeezed against a rough surface in a 


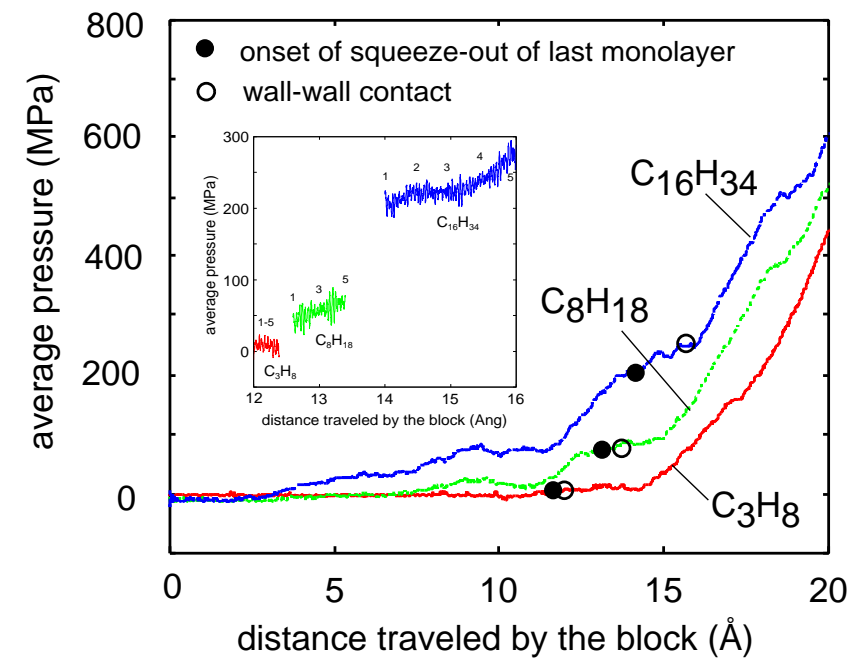

FIG. 5: The average pressure as a function of the chain length. The squeezing velocity is $1 \mathrm{~m} / \mathrm{s}$. The black dots correspond to the pressures and distances at which the squeeze out of the last monolayer is initiated. The open circles show the point of wall-wall contact, which refer to the state shown in the last images (image 5) in Fig. 4. The insert shows the detailed squeeze-out pressures of the last monolayer for the three systems. The numbers 1-5 correspond to images 1-5 in Fig. 4.

fluid. In this case the trapped fluid will effectively smooth the surface and reduce the viscoelastic contribution to the friction [30].

In Fig. 7 we show the pressure at the squeeze out of the last monolayer, as a function of the chain length. Results for squeezing, and for squeezing and sliding, are shown. The squeeze-out pressure refer to the squeeze-out state shown by the last images (image 5 ) in Fig. 4. The solid line is the dependency of the pressure on the chain length $N$ when the lubricant film is in the $2 \mathrm{D}$ fluid-like state. Note that the dashed fit-line extrapolate to zero pressure before reaching $N=1$, indicating two regimes of different behavior associated with 2D fluid-like and solid-like monolayers, respectively.

Fig. 8 shows the pressures at initial contact between the block and the substrate as a function of the temperature. The distance traveled by the block at this contact is also shown. Notice that the lowest squeeze-out pressures and the shortest distances are found when the alkanes are close to or above their boiling point. On the other hand the squeeze-out pressures are about the same $(\sim$ $200 \mathrm{MPa}$ ) at the melting point. The discussed physical properties of the alkanes can be found in table I. Under ambient conditions, in presence of oxygen, the $\mathrm{C}_{8} \mathrm{H}_{18}$ and $\mathrm{C}_{16} \mathrm{H}_{34}$ systems would auto-ignite at temperature above $500 \mathrm{~K}$. In our MD simulations oxygen is absent so this reaction scheme is not an issue here.
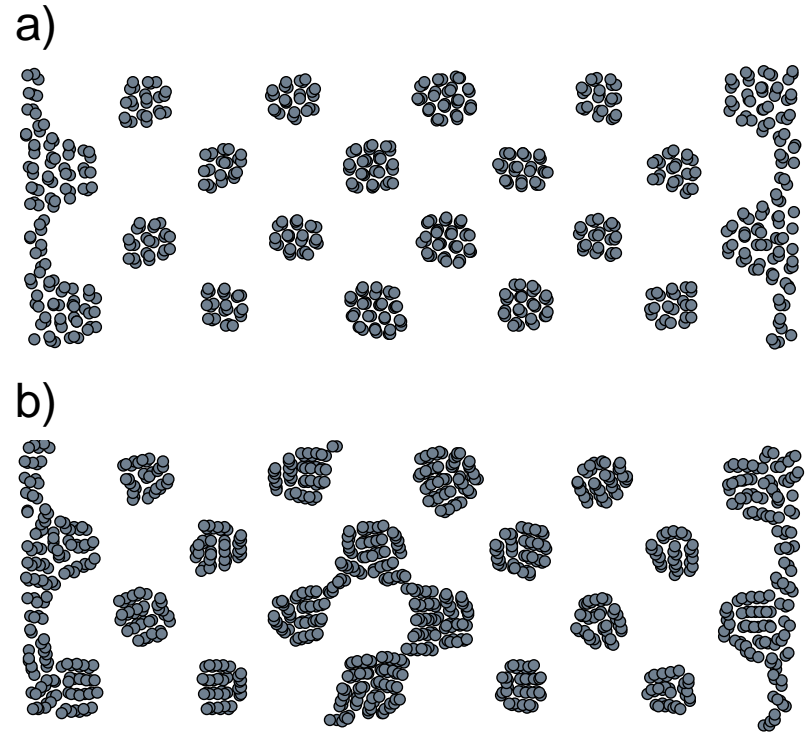

C)

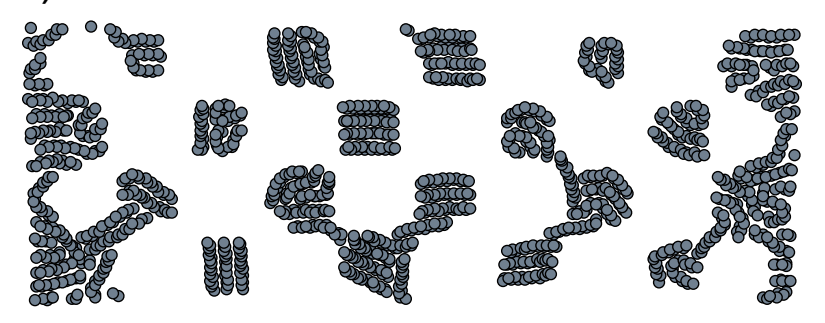

FIG. 6: Snapshot pictures of the $\mathrm{C}_{3} \mathrm{H}_{8}, \mathrm{C}_{8} \mathrm{H}_{18}$ and $\mathrm{C}_{16} \mathrm{H}_{34}$ systems when the upper surface of the block has moves $10 \AA$ towards the substrate, after the point where the substrate and the block, in the absence of the lubricant, first made contact.

\section{DISCUSSION}

One remarkable observation made above is the small pressure needed to squeeze-out the $\mathrm{C}_{3} \mathrm{H}_{8}$ lubricant as compared to the $\mathrm{C}_{16} \mathrm{H}_{34}$. We will now show that this is due to the fact that at room temperature the $\mathrm{C}_{3} \mathrm{H}_{8}$ lubricant is in a $2 \mathrm{D}$ liquid-like state, while the $\mathrm{C}_{8} \mathrm{H}_{18}$ and $\mathrm{C}_{16} \mathrm{H}_{34}$ films are in a $2 \mathrm{D}$ solid-like state.

The corrugated substrate surface can locally, in the vicinity of the top of an asperity, be approximated by a spherical (or elliptic) bump with the (mean) curvature $1 / R=q_{x} q_{y} h_{0}$. Let us assume that the lubricant monolayer is in a $2 \mathrm{D}$ liquid-like state.

We neglect the elastic deformations of the solid walls, and also the direct interaction between the asperity (here sphere) and the substrate (which requires that the minimum separation $u$ (see Fig. 9) between the solid walls is not too small). We now consider how the $2 \mathrm{D}$ fluid is squeezed out from an asperity contact region.

Fig. 9 shows a sphere (radius $R$ ) pushed against an adsorbate layer where the adsorbates are treated as spheres with the radius $r_{0}$. Let $u$ be the shortest separation between the sphere and the substrate. From Fig. 9 we 


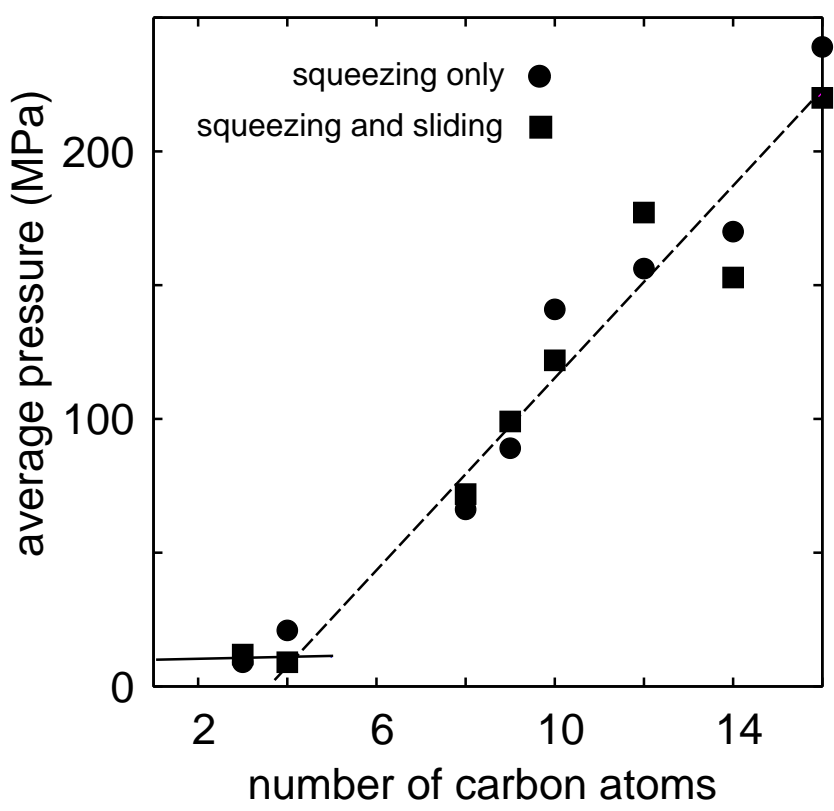

FIG. 7: The average pressure at the squeeze-out of the last monolayer as a function of the chain length $N$. Results for squeezing, and for squeezing and sliding, are shown. The sliding and squeezing velocities are both $1 \mathrm{~m} / \mathrm{s}$. The lines are guides for the eye.

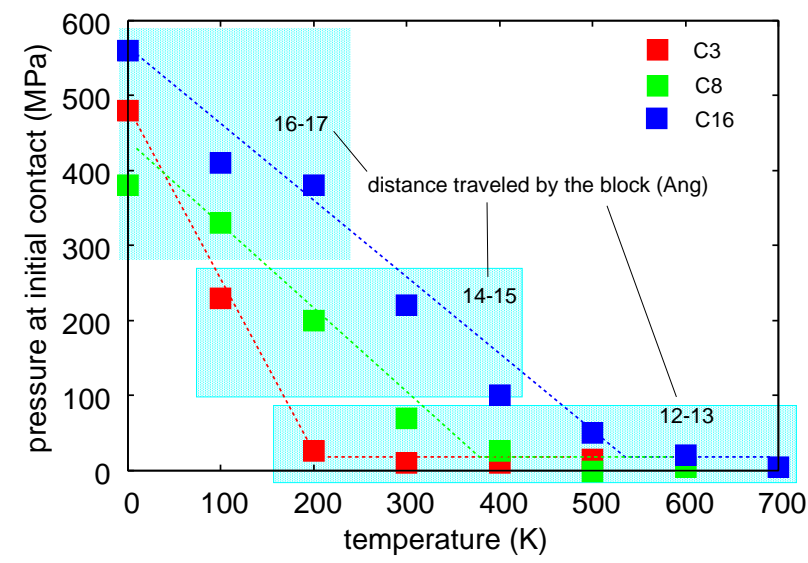

FIG. 8: Initial contact pressures of $\mathrm{C}_{3} \mathrm{H}_{8}, \mathrm{C}_{8} \mathrm{H}_{18}$ and $\mathrm{C}_{16} \mathrm{H}_{34}$ as a function of the temperature. The distance traveled by the block at the squeeze-out is also shown. The dotted lines are guides for the eye.

get

$$
\begin{gathered}
\left(R+r_{0}\right) \cos \theta+r_{0}=R+u \\
r=\left(R+r_{0}\right) \sin \theta
\end{gathered}
$$

Combining these equations gives the surface area $A$ without adsorbates

$$
A=\pi r^{2}=\pi\left[4 R r_{0}+u\left(2 r_{0}-2 R-u\right)\right]
$$

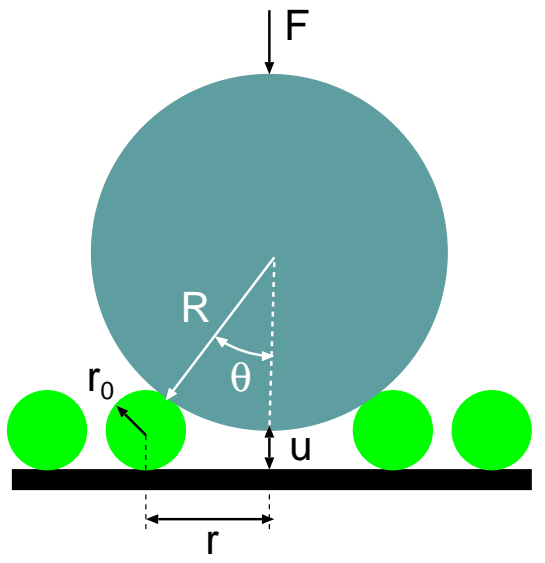

FIG. 9: A hard asperity, here represented as a sphere, penetrating an adsorbed film of molecules (green spheres). (a) block

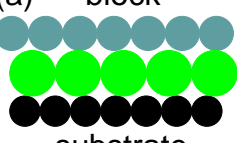

substrate

the work = S A (b)

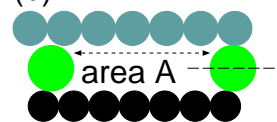

lubricant

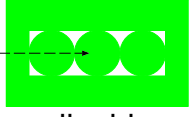

liquid
FIG. 10: The spreading pressure $S$ is the energy per unit surface area to move a patch (surface area $A$ ) of the lubricant film from the region between the block and the substrate, to the lubricant fluid outside of the contact region. This energy can be obtained by subtracting the calculated Lennard-Jones interaction energy between the patch of molecules and the surrounding in the two different configurations (between the walls and in the fluid).

Where $r_{0}$ is negligible compared to $\mathrm{r}$. The work necessary to remove the adsorbates from this area is $U=S A$, where $S$ the spreading pressure. Thus the normal force acting on the sphere is

$$
F=-\frac{d U}{d u}=2 \pi S\left(R+u-r_{0}\right)
$$

If we assume that $u-r_{0}$ is small compared to $R$ this gives $F \approx 2 \pi S R$, corresponding to the pressure

$$
p=\frac{F}{\lambda_{x} \lambda_{y}}=\frac{q_{x} q_{y} F}{(2 \pi)^{2}}=\frac{S q_{x} q_{y} R}{2 \pi}=\frac{S}{2 \pi h_{0}}
$$

TABLE I: Some Physical properties of the investigated alkanes [31]

\begin{tabular}{|l|l|l|}
\hline & Melting point $(\mathrm{K})$ & Boiling point $(\mathrm{K})$ \\
\hline $\mathrm{C}_{3} \mathrm{H}_{8}$ & 85 & 231 \\
\hline $\mathrm{C}_{8} \mathrm{H}_{18}$ & 216 & 398 \\
\hline $\mathrm{C}_{16} \mathrm{H}_{34}$ & 291 & 560 \\
\hline
\end{tabular}

In the present case $h_{0}=5 \AA$, and using the estimated spreading pressure (see Fig. 10) for the $\mathrm{C}_{3} \mathrm{H}_{8}$ 
lubricant, $S \approx 0.2 \mathrm{~J} / \mathrm{m}^{2}$, gives the pressure needed to squeeze out the lubricant: $p \approx 67 \mathrm{MPa}$. The average pressure at the squeeze-out of the last monolayer from the molecular dynamics calculations is $p_{0} \approx 10 \mathrm{MPa}$. If we assume the whole applied normal force is acting on the 4 Hertz's contact regions, then the average pressure acting in one of the $\lambda_{x} \times \lambda_{y}$ contact regions will be $p=(13 \times 2 / 4) p_{0}=65 \mathrm{MPa}$ i.e., very close to the calculated pressure. Thus this simple model not only predicts the correct squeeze-out pressure but also explain why $p$ is approximately constant during the initial phase of the squeeze-out of the last monolayer.

Let us now consider the fluid squeeze-out for the longer chain alkane systems. In these cases the adsorbed monolayer film is in a solid-like state with small domains of ordered alkane chains, which are likely to be pinned by the substrate potential, even if no commensurate adsorbate layer is formed globally. If a transversal force acts on the adsorbate film from a nano-scale asperity (as in Fig. 9), only a small lateral displacement of the adsorbed molecules will take place, unless the parallel force is large enough to move the film over the top of the (local) pinning barriers. Thus, depending on the pinning barrier height, the onset of the squeeze-out could be at much higher applied pressure than when the film is in a $2 \mathrm{D}$ liquid-like state. Furthermore, when the monolayer is in a solid-like state the squeeze-out maybe unrelated to the concept of spreading pressure.

In Ref. [2] we found that the number of carbon atoms per unit surface area in the monolayer film increases with the alkane chain length as $c \approx(9.55+0.62 N) \mathrm{nm}^{-2}$, where $N$ is the number of carbon atoms in the chain. Assuming in a fist approximation that the spreading pressure is proportional to the concentration $c$ we get when the alkane film is in the $2 \mathrm{D}$ liquid-like state the scaling $p_{0} \approx(10 \mathrm{MPa}) \times(0.84+0.054 N)$. The solid line in Fig. 7 shows this function.

The fluid squeeze-out mechanism presented above assumes that the surfaces have short wavelength (nanoscale) surface roughness. In Ref. [2] the surfaces were smooth and in this case a very different squeeze-out mechanism prevail, involving the nucleation of a small circular open area in the lubrication film, and the bending of the solid walls into the cavity. This process gives rise to much larger squeeze-out pressures than found above, in agreement with the molecular dynamics results presented in Ref. [2] where the squeeze-out pressure increased nearly linearly with the chain length from $\approx 350 \mathrm{MPa}$ for $\mathrm{C}_{3} \mathrm{H}_{8}$ to $\approx 800 \mathrm{MPa}$ for $\mathrm{C}_{16} \mathrm{H}_{34}$.

We have verified the above calculations by extending our Molecular Dynamics calculations. We have squeezed the alkanes at temperatures from OK to above their bulk boiling points (see tabel I). Fig. 8 shows that the initial squeeze-out pressures are $\sim 10 \mathrm{MPa}$ for all the systems when they are in the $2 \mathrm{D}$ liquid state. This is realised at the bulk boiling point of the alkanes. In addition to this, the distance traveled by the block at squeeze-out is similar for all the systems in the $2 \mathrm{D}$ liquid state. As the temperature is decreased we observe that the squeezeout pressure, in average, is higher for the longer alkanes, which is in good accordance with our discussion regarding the increased ability to form crystallin domains for longer alkanes. It is interesting to observe that the squeeze-out pressure is about the same for all the systems at their melting point. At this phase transition the molecules show the same resistance to squeeze-out regardless of their length. This analysis shows that all the systems behave similarly at their melting and boiling points. It is therefore less crucial to calculate the squeeze-out pressures of C5 to C7 in Fig. 7 .

The picture presented above for the squeeze-out is valid for any type of lubrication molecules. Fatty acids are often added to the base oil of lubrication fluids. These molecules form grafted monolayer films on iron oxide surfaces and reduce the friction and wear. In a beautiful study Rabinowicz and Tabor[32] performed friction and wear experiments (and electric contact resistance measurements) on iron surfaces lubricated by palmic acid. They observed low friction and negligible wear below $T=T_{0} \approx 110^{\circ} \mathrm{C}$, but a rapid increase in the friction and wear above this temperature. This was interpreted as resulting from melting of the $2 \mathrm{D}$ grafted monolayer film at $T \approx T_{0}$, and rapid squeeze-out of the fluid boundary lubricant film. This picture is consistent with our theory and simulation results.

\section{SUMMARY AND CONCLUSIONS}

The squeeze-out mechanism of hydrocarbon lubricants between a smooth curved surface and a flat nanocorrugated one has been been investigated by molecular dynamics simulations. The pressure required for squeezeout increases drastically with increasing of the hydrocarbon molecular length. It is remarkable that a pressure more than 20 times larger is required for squeezing out $\mathrm{C}_{16} \mathrm{H}_{34}$ than for $\mathrm{C}_{3} \mathrm{H}_{8} . \mathrm{C}_{3} \mathrm{H}_{8}$ is fluid-like at the temperature chosen for the simulations $(300 \mathrm{~K})$. This means high mobility of the molecules on the surfaces which results in squeeze-out at low pressures. The longer hydrocarbons $\left(\mathrm{C}_{8} \mathrm{H}_{18}\right.$ and $\left.\mathrm{C}_{16} \mathrm{H}_{34}\right)$ form domains of equally oriented molecules that render nucleation more difficult and thereby increase the pressure required for squeezeout of the lubricant. By varying the temperature during squeeze-out we can observe that all the investigated alkanes show very low initial contact pressures at about and above their bulk boiling point which indicates that they are in the same 2D liquid state at these temperatures. Regardless of the applied pressure, hydrocarbons are trapped in the valleys of the surface roughness. Longer molecules also form hydrocarbon bridges between the valleys which supposedly decrease the wear of the surfaces in practical applications.

We find good accordance between our simulations and experiments showing that longer hydrocarbons are better boundary lubricants than shorter ones. 


\section{Acknowledgments}

I.M.S. acknowledges support from IFF, FZ-Jülich, hospitality and help of the staff during his research visits. The research work was performed within a ReinhartKoselleck project funded by the Deutsche Forschungsgemeinschaft (DFG). We would like to thank DFG for the project support under the reference German Research Foundation DFG-Grant: MU 1225/36-1. The research work was also supported by the DFG-grant: PE 807/101. This work is supported in part by COST Action MP1303.
[1] B.N.J. Persson and F. Mugele, Squeeze-out and wear: Fundamental principles and applications Journal of Physics: Condensed matter 16, R295 (2004).

[2] I M Sivebaek, V N Samoilov and B N J Persson: Squeezing molecular thin alkane lubrication films between curved solid surfaces with long-range elasticity: Layering transitions and wear, Journal of Chemical Physics 119, 2314 (2003)

[3] I. M. Sivebaek, V. N. Samoilov, and B. N. J. Persson: Frictional properties of confined polymers Eur. Phys. J. E. 27, 37 (2008)

[4] I. M. Sivebaek, V. N. Samoilov, and B. N. J. Persson: Velocity dependence of friction of confined hydrocarbons Langmuir 26, 8721 (2010)

[5] I. M. Sivebaek, V. N. Samoilov, and B. N. J. Persson: Effective viscosity of confined hydrocarbons Phys. Rev. Lett. 108, 1 (2012)

[6] I. M. Sivebaek and B. N. J. Persson: Shearing Nanometer-Thick Confined Hydrocarbon Films: Friction and Adhesion Tribol. Lett.. 62, 5 (2016)

[7] D. Savio, N. Fillot, and P. Vergne: A molecular dynamics study of the transition from ultra-thin film lubrication toward local film breakdown Tribol. Lett. 50, 207 (2013)

[8] N. N. Gosvami and S. J. OShea: Nanoscale Trapping and Squeeze-Out of Confined Alkane Monolayers Langmuir 31, 12960 (2015)

[9] M.-D. Krass, N. N. Gosvami, R. Carpick, M. H. Müser, and R. Bennewitz: Dynamic shear force microscopy of nanometer-confined hexadecane layers J. Phys. Condens. Matter 28, 1 (2016)

[10] L. T. W. Lim, A. T. S. Wee, and S. J. OShea: Temperature dependence of solvation forces as measured in atomic force microscopy J. Chem. Phys. 1301 2009)

[11] N. N. Gosvami, S. K. Sinha, W. Hofbauer, and S. J. OShea: Solvation and squeeze out of hexadecane on graphite J. Chem. Phys. 126, (2007)

[12] A. M. Homola, J. N. Israelachvili, P. M. McGuiggan and M. L. Gee", Fundamental experimental studies in tribology. The transition from 'interfacial' friction of undamaged molecularly smooth surface to 'normal' friction with wear Wear 136, 65 (1990)

[13] F. Mugele and M. Salmeron: Dynamics of Layering Transitions in Confined Liquids Physical Review Letters 84 , 5796 (2000)

[14] T. Becker and F. Mugele: Collapse of molecularly thin lubricant layers between elastic substrates Journal of Physics: Condenced Matter 15, S321 (2003)

[15] Standard Test Method for Evaluating Lubricity of Diesel Fuels by the High-Frequency Reciprocating Rig (HFRR) Annual Book of ASTM Standards: D6079-97 5.03 1355$1360(1998)$
[16] Diesel Fuel - Assessment of Lubricity using the HighFrequency Reciprocating rig (HFRR) ISO Standards ISO 12156-1 and 2 (1997)

[17] I. M. Sivebaek and S. C. Sorenson: Dimethyl Ether ( DME ) Assessment of Lubricity Using the Medium Frequency Pressurised Reciprocating Rig Version 2 ( $M F$ PRR2) SAE, (2000)

[18] B. N. J. Persson and P. Ballone: Squeezing Lubrication Films: Layering Transition for Curved Solid Surfaces with Long-range Elasticity, Journal of Chemical Physics 112, 9524 (2000)

[19] U. Tartaglino, I. M. Sivebaek, B. N. J. Persson, and E. Tosatti: Impact of molecular structure on the lubricant squeeze-out between curved surfaces with long range elasticity J. Chem. Phys. 125, (2006)

[20] W.L. Jorgensen, J.D. Madura and C.J. Swenson: Optimized Intermolecular Potential Functions for Liquid Hydrocarbons, J. Am. Chem. Soc. 106, 6638 (1984).

[21] D.K. Dysthe, A.H. Fuchs and B. Rousseau: Fluid transport properties by equilibrium molecular dynamics. III. Evaluation of united atom interaction potential models for pure alkanes, J. Chem. Phys. 112, 7581 (2000).

[22] T. K. Xia and J. Ouyang and M. W. Ribarsky and U. Landman: Interfacial alkane films Physical Review Letters 69, 1967 (1992).

[23] S. Eder, A. Vernes and G. Betz Methods and numerical aspects of nanoscopic contact area estimation in atomistic tribological simulations Computer Physics Communications 185, 217 (2014)

[24] I. Szlufarska, M. Chandross and R.W. Carpick Recent advances in single-asperity nanotribology J. Phys. D: Appl. Phys. 41123001 (2008)

[25] B.Q. Luan, S. Hyun, F. Molinari, N. Bernstein and M.O. Robbins Multiscale modeling of two-dimensional contacts PHYSICAL REVIEW E 74, 046710 (2006)

[26] M.H. Müser Single-asperity contact mechanics with positive and negative work of adhesion: Influence of finiterange interactions and a continuum description for the squeeze-out of wetting fluids Beilstein J. Nanotechnol. 5, 419 (2014)

[27] B.N.J. Persson, O. Albohr, G. Heinrich and H. Ueba, Crack propagation in rubber-like materials JOURNAL OF PHYSICS-CONDENSED MATTER 17, R1071 (2005)

[28] A. Jabbarzadeh, P. Harrowell and R.I. Tanner, Crystal Bridge Formation Marks the Transition to Rigidity in a Thin Lubrication Film Phys. Rev. Lett. 96, 206102 (2006)

[29] A. Jabbarzadeh and R.I. Tanner Flow-Induced Crystallization: Unravelling the Effects of Shear Rate and Strain Macromolecules 43, 8136 (2010) 
[30] B.N.J. Persson, U. Tartaglino, O. Ahlbor and E. Tosatti, Rubber friction on wet and dry road surfaces: The sealing effect PHYSICAL REVIEW B 71, 035428 (2005)

[31] CRC Handbook of Chemistry and Physics Editor-inChief: W. M. Haynes 97th Edition (2016-2017) CRC Press Taylor \& Francis Group
[32] R. Rabinowicz and D. Tabor, Proc. Roy. Soc. METALLIC TRANSFER BETWEEN SLIDING METALS - AN AUTORADIOGRAPHIC STUDY (London) A 208, 455 (1951). 